\title{
The Habitable Exoplanet Observatory (HabEx)
}

B. Scott Gaudi, Sara Seager, Bertrand Mennesson, Alina Kiessling, Keith Warfield

B. Scott Gaudi, Sara Seager, Bertrand Mennesson, Alina Kiessling, Keith Warfield, "The Habitable Exoplanet Observatory (HabEx)," Proc. SPIE 11115, UV/Optical/IR Space Telescopes and Instruments: Innovative Technologies and Concepts IX, 111150M (9 September 2019); doi: 10.1117/12.2530036

SPIE Event: SPIE Optical Engineering + Applications, 2019, San Diego, California, United States 


\author{
The Habitable Exoplanet Observatory (HabEx) \\ B. Scott Gaudi*a, Sara Seager ${ }^{\mathrm{b}}$, Bertrand Mennesson ${ }^{\mathrm{c}}$, Alina Kiessling ${ }^{\mathrm{c}}$, Keith Warfield ${ }^{\mathrm{c}}$, \\ and the HabEx Study Team \\ ${ }^{\text {a }}$ Department of Astronomy, The Ohio State University, $140 \mathrm{~W} .18^{\text {th }}$ Ave., Columbus, OH 43210 \\ ${ }^{b}$ Department of Earth, Atmospheric and Planetary Sciences, Department of Physics, Massachusetts \\ Institute of Technology, 77 Massachusetts Ave., Cambridge, MA 02139 \\ 'Jet Propulsion Laboratory, California Institute of Technology, 4800 Oak Grove Drive, Pasadena, \\ CA 91109
}

\begin{abstract}
The Habitable Exoplanet Observatory, or HabEx, has been designed to be the Great Observatory of the 2030s. For the first time in human history, technologies have matured sufficiently to enable an affordable space-based telescope mission capable of discovering and characterizing Earthlike planets orbiting nearby bright sunlike stars to search for signs of habitability and biosignatures. Such a mission can also be equipped with instrumentation that will enable broad and exciting general astrophysics and planetary science not possible from current or planned facilities. HabEx is a space telescope with unique imaging and multi-object spectroscopic capabilities at wavelengths ranging from ultraviolet (UV) to near-IR. These capabilities allow for a broad suite of compelling science that cuts across the entire NASA astrophysics portfolio. HabEx has three primary science goals: (1) Seek out nearby worlds and explore their habitability; (2) Map out nearby planetary systems and understand the diversity of the worlds they contain; (3) Enable new explorations of astrophysical systems from our own solar system to external galaxies by extending our reach in the UV through near-IR. This Great Observatory science will be selected through a competed GO program, and will account for about $50 \%$ of the HabEx primary mission. The preferred HabEx architecture is a $4 \mathrm{~m}$, monolithic, off-axis telescope that is diffractionlimited at $0.4 \mu \mathrm{m}$ and is in an L2 orbit. HabEx employs two starlight suppression systems: a coronagraph and a starshade, each with their own dedicated instrument.
\end{abstract}

Keywords: decadal, exoplanets, biosignatures, high contrast imaging, galaxy formation and evolution, coronagraph, starshade

*gaudi.1@osu.edu; phone 1614 292-1914

\title{
1. INTRODUCTION
}

For the first time in human history, technologies have matured sufficiently to enable an affordable space-based telescope mission capable of discovering and characterizing habitable planets like Earth orbiting nearby bright sunlike stars. Such an observatory can be equipped with instruments that provide a wide range of capabilities, enabling unique science not possible from ground-based facilities. This science is broad and exciting, ranging from new investigations of our own solar system, to understanding the life cycle of baryons and its impact on the formation and evolution of galaxies, to addressing fundamental puzzles in cosmology.

The Habitable Exoplanet Observatory, or HabEx, has been designed to be the Great Observatory of the 2030s, a successor to the Hubble Space Telescope (HST) with enhanced capabilities and community involvement through a competed and funded Guest Observer (GO) program. This GO program - which shall represent $50 \%$ of HabEx's prime 5year mission - will include competed novel observations, parallel and serendipitous observations, and archival research. After HabEx's 5-year primary mission, HabEx is capable of undertaking an extended mission of at least five additional years without reservicing, during which the GO program would represent $100 \%$ of observing time. HabEx is a space-based 4-meter diameter telescope with ultraviolet (UV), optical, and near-infrared (near-IR) imaging and spectroscopic capabilities, replacing and enhancing those lost at the end of HST's lifetime.

\section{HABEX SCIENCE}

HabEx has three driving science goals during its 5-year primary mission (Figure 1).

UV/Optical/IR Space Telescopes and Instruments: Innovative Technologies and Concepts IX, edited by Allison A. Barto, James B. Breckinridge, H. Philip Stahl, Proc. of SPIE Vol. 11115, 111150M · (c) 2019 SPIE CCC code: $0277-786 X / 19 / \$ 21 \cdot$ doi: $10.1117 / 12.2530036$ 


\subsection{HabEx will seek out nearby worlds and explore their habitability.}

A pervasive and fundamental human question is: Are we alone? Astronomy has recast this elemental inquiry into a series of questions: Are there other Earths? Are they common? Do any have signs of life? Space-based direct imaging above the blurring effects of our atmosphere is the only way to discover and study exo-Earths candidates (EECs) in reflected light, i.e., the only way to take detect and spectra of Earth-sized planets in Earth-like orbits in reflected light (at near-UV, optical, and near-IR wavelengths) about sunlike (F, G, and Ktype) stars.

With unparalleled high-contrast direct imaging capabilities, HabEx will spectrally characterize dozens of rocky worlds, including validating (obtaining orbits and 0.3$1 \mu \mathrm{m}$ spectra) of $\sim 8$ EECs (Figure 1). It will also detect and characterize over a hundred larger planets around mature stars (Figure 2). With five visits to each system, using the coronagraph HabEx will measure the orbits of EECs to a few percent in the semimajor axis and inclination, and will determine the eccentricity to $\sim 0.02$, allowing a robust determination of whether or not the EEC resides in the

To seek nearby worlds and explore their habitability.

\section{To map out nearby planetary} systems and understand the diversity of worlds they contain.
Enable new explorations of astrophysical systems from our solar system to galaxies and the universe by extending our reach in the UV through near-IR.

Habitable Zone. Of particular interest for investigations of EECs, HabEx will be sensitive to Rayleigh scattering, water vapor $\left(\mathrm{H}_{2} \mathrm{O}\right)$, molecular oxygen $\left(\mathrm{O}_{2}\right)$ and ozone $\left(\mathrm{O}_{3}\right)$. It will detect all three gases down to column densities as low as $1 \%$ of modern Earth levels. In addition, HabEx will detect other atmospheric gases for context, such as methane and carbon dioxide, if they have concentrations higher than modern Earth. For our nearest neighbors, HabEx will also search for evidence of surface liquid water oceans on exo-Earth candidates by searching for specular reflection, or glint.

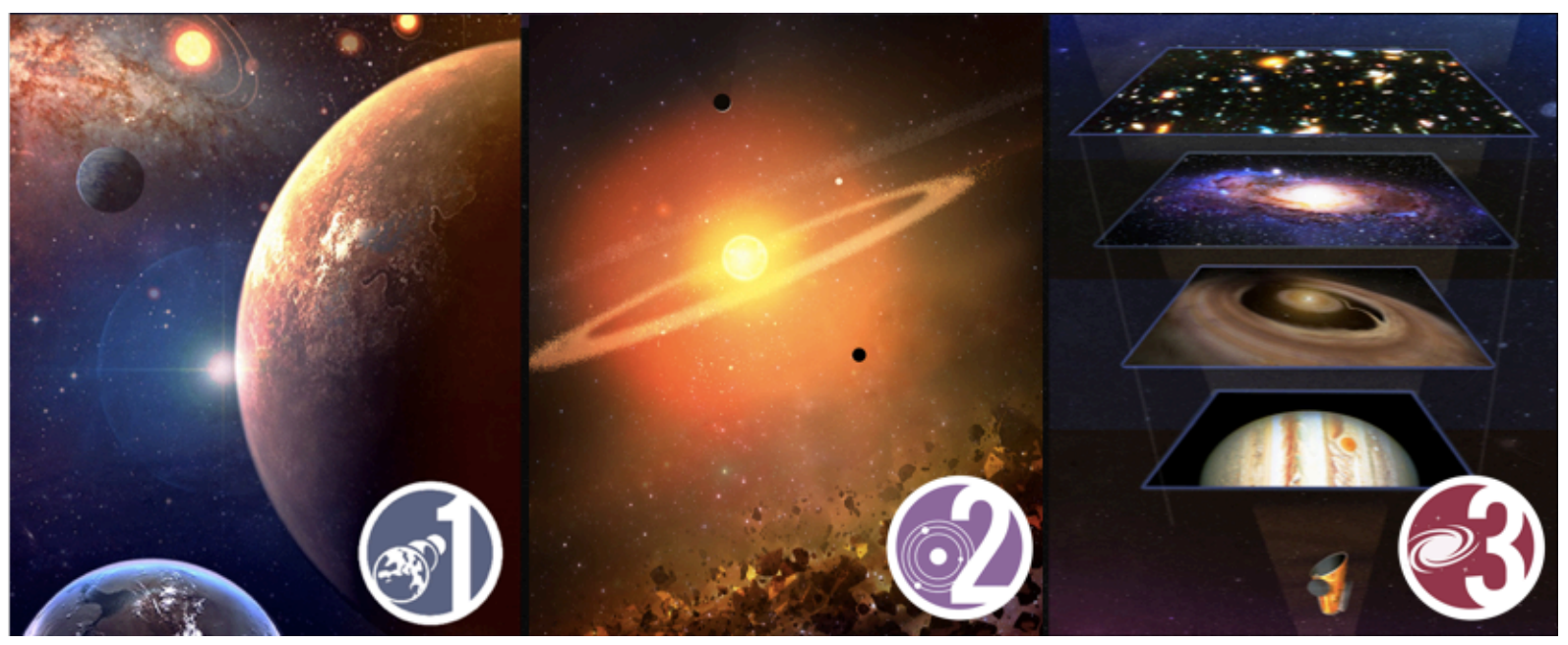

Figure 1. The HabEx Observatory has three science goals: 1. To seek out nearby worlds and explore their habitability, 2. To map out nearby planetary systems and understand the diversity of the worlds they contain, and 3. Enable new explorations of astrophysical systems from our own solar system to galaxies and the universe by extending our reach in the UV through the near-IR. 

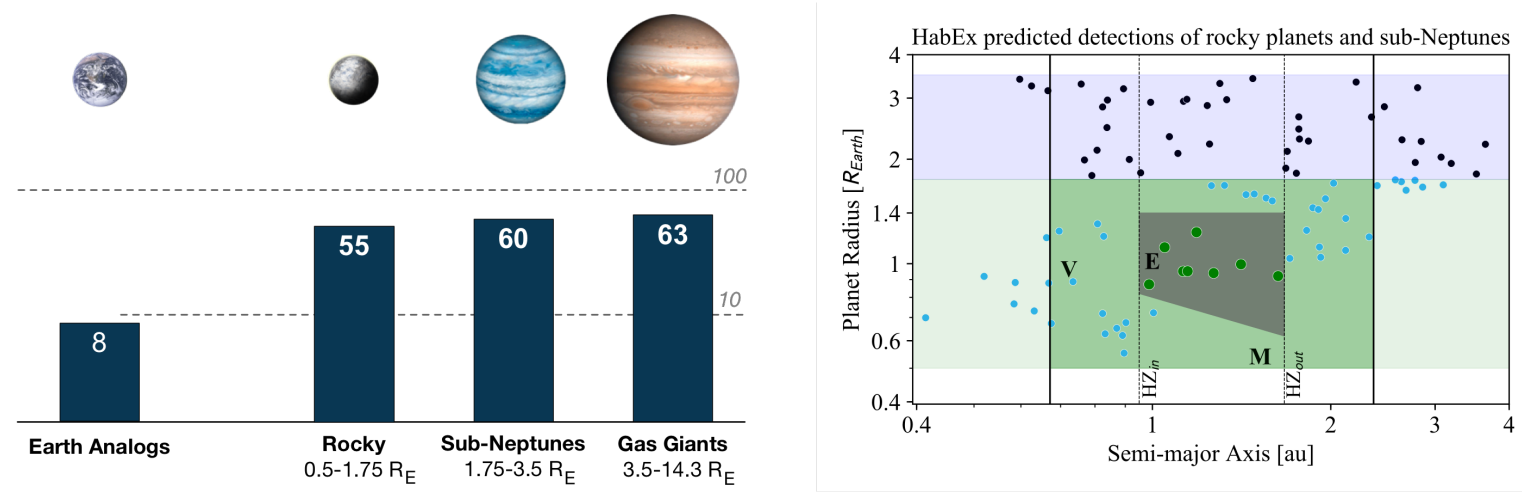

Figure 2. Left panel (note log scale): Under the design reference mission and survey strategy, assuming nominal occurrence rates [1], HabEx will detect over 150 exoplanets with a diversity of sizes and temperatures. It will obtain broad spectra (from at least from $0.3-1 \mu \mathrm{m}$ ) of the majority of these planets, including $\sim 37$ rocky planets proximate to the HZ (Right panel, dark green region), and will get orbits and spectra of $\sim 8$ planets with radii and separations consistent with the adopted conservative definition of exo-Earth candidates (EECs, Right panel, grey shaded region). HabEx will empirically constrain the HZ region concept, both in terms of planet separations and radii. Right panel: Zooming into HabEx small planet characterization space close to the HZ. Note on the right panel the semi-major axis boundaries are for a solar twin. For other host stars, they have been scaled to maintain a constant bolometric insolation.

\subsection{HabEx will map out nearby planetary systems and understand the diversity of the worlds they contain.}

With high-contrast $12 \times 12 \operatorname{arcsec}^{2}$ (equivalent to $36 \times 36 \mathrm{AU}^{2}$ at a distance of $3 \mathrm{pc}$ ) observations using the starshade, HabEx will be the first observatory capable of providing nearly complete "family portraits" of our nearest neighbors. HabEx will characterize full individual planetary systems, including exoplanet analogs to Earth, Saturn and Jupiter, and analogs to the zodiacal and Kuiper dust belts. For many of these planets, HabEx will not only obtain multi-epoch broadband spectra from 0.3-1.0 $\mu \mathrm{m}$ (and in some cases from 0.2-1.8 $\mu \mathrm{m}$ ), but for those with periods of $<10$ years, will also determine the orbital parameters to a typical precision of $<5 \%$ on the inclination, $25 \%$ on the semimajor axis, and measure the eccentricity to an uncertainty of 0.1 . HabEx is also expected to find and spectrally characterize a diversity of planetary systems that bear little resemblance to our system, including those with worlds that have no analogs in our solar system, but are known to common in other planetary systems, including super-Earths and sub-Neptunes (Figure 2). In general, given that HabEx's requirements are set by the characterization of EECs, the spectra of all planets that are brighter than an EEC will have much higher signal-to-noise ratio.

Discoveries of nearby planetary systems will provide detailed architectures, addressing open topics ranging from planetary system formation, planetary migration, and to the role of gas giants in the delivery of water to inner system rocky worlds. HabEx will test theories on planetary diversity, investigate planet-disk interactions, and place our solar system into detailed context for the first time

\subsection{HabEx will enable new explorations of astrophysical systems from our own solar system to galaxies and the universe by extending our reach in the UV through near-IR.}

HabEx will be NASA's Great Observatory in the 2030s. Observing with a large aperture from above the Earth's atmosphere in an era when neither the Hubble Space Telescope (HST) nor the James Webb Space Telescope (JWST) are operational, HabEx will provide the highest-resolution images yet obtained at UV and optical wavelengths (Figure 5). HabEx will also provide an ultra-stable platform and access to wavelengths inaccessible from the ground.

These capabilities allow for a broad suite of unique, compelling science that cuts across the entire NASA astrophysics portfolio and includes topics as diverse as the life cycle of baryons, the sources of the metagalactic ionizing background, the origins of the elements from the first generations of stars and supernovae, the local expansion rate of the universe, dark matter models, the formation of Galactic globular clusters, the atmospheres of transiting exoplanets via transit spectroscopy, interactions between the Sun and the giant planets in our solar system, and the structure of protoplanetary transition disks. 

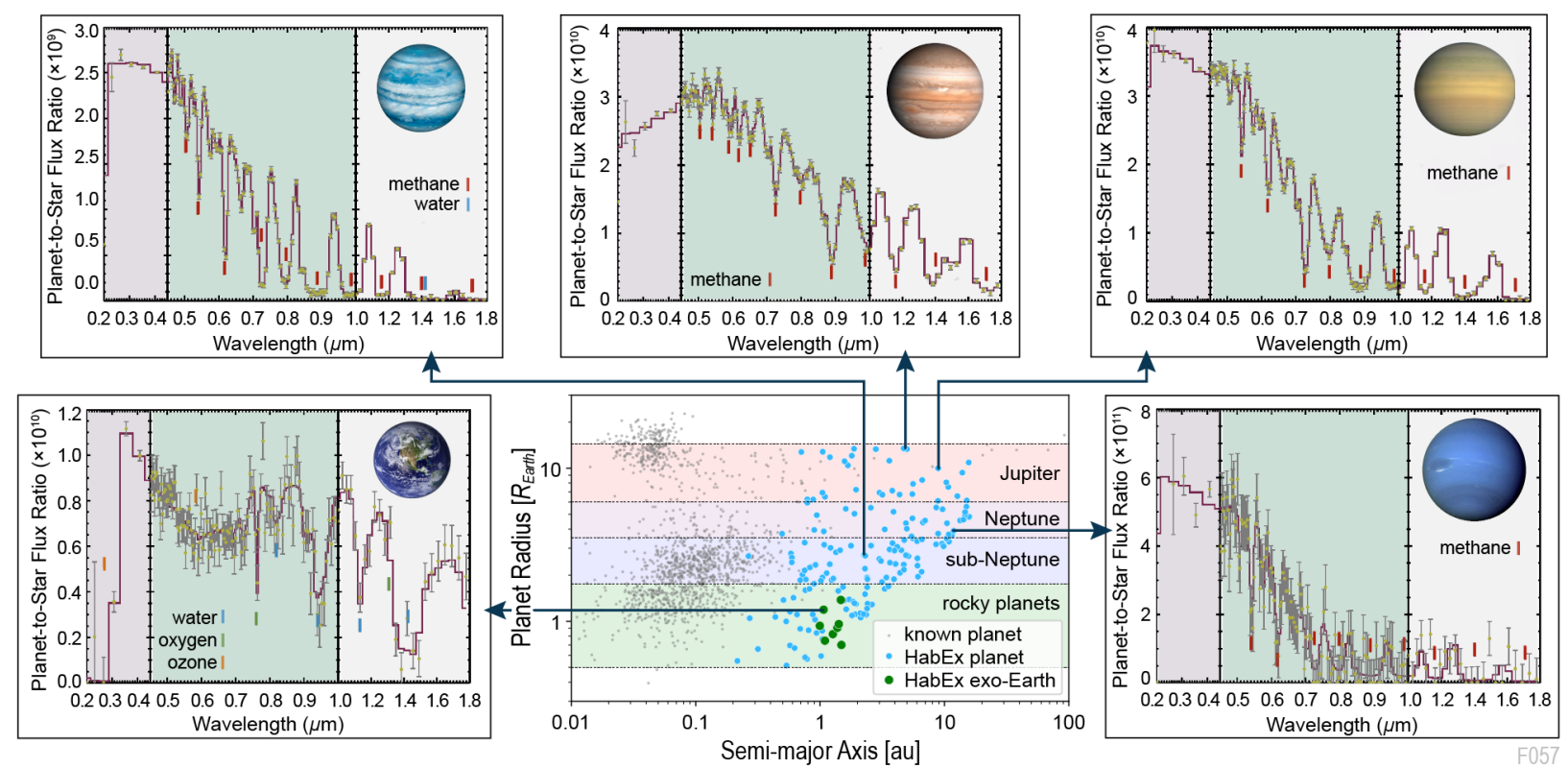

Figure 3. HabEx will discover and characterize over 150 new exoplanets (cyan points), from small exo-Earths candidates (green points) to gas giants, populating previously unexplored regions of parameter space, including planets that have no analogues in our solar system. The majority of these worlds will be well-characterized, with relatively high signal-to-noise ratio ( $\mathrm{SNR} \geq 10)$, resolution $\mathrm{R} \sim 140$ spectra from $0.45-1.0 \mu \mathrm{m}$. By moving the starshade further away from telescope, HabEx will obtain crude spectra with resolution $\mathrm{R} \sim 7$ in the UV channel from $0.20-0.45 \mu \mathrm{m}$, and by moving the starshade closer from the telescope, HabEx will obtain spectra with resolution R 40 in the near-IR channel from 0.95-1.8 microns. Thus, HabEx can obtain complete spectral coverage of many of the planets discovered from $0.2-1.8 \mu \mathrm{m}$. Using multiple visits with the coronagraph, HabEx will also obtain reasonably precise orbits (inclinations measured to $<5 \%$, semimajor axes to $25 \%$, eccentricities with an uncertainty of 0.1 ) for those with periods of less than roughly 10 years and eccentricities less than roughly 0.3 within the nominal 5 -year mission lifetime. These orbital uncertainties improve significantly for shorter period orbits, particularly those with periods less than the mission lifetime. Thus, HabEx can obtain "family portraits" of a diversity of worlds in nearby planetary systems.

Of course, we cannot know which of the scientific questions that motivate HabEx's GO program as outlined here will still be relevant in the 2030s. However, by designing HabEx to have capabilities that significantly extend and enhance those of any current or planned mission, we can rely on the community's imagination and future priorities to maximize the science return of the mission.

\section{BASELINE HABEX IMPLEMENTATION}

The HabEx Observatory design utilizes an off-axis, monolithic primary $4 \mathrm{~m}$ diameter telescope, diffraction-limited at $0.4 \mu \mathrm{m}$, launched on an SLS 1B launch vehicle to an Earth-Sun L2 orbit, and a 52m starshade spacecraft, separately launched on a Falcon Heavy launch vehicle, also to an Earth-Sun L2 orbit (see [2] for more details). The nominal distance between the spacecraft and the starshade is $\sim 76,600 \mathrm{~km}$, but the starshade can be moved closer or further away from the telescope to increase the range of wavelengths covered by the telescope and starshade.

HabEx has two starlight suppression systems: a coronagraph and a starshade, each with their own dedicated instruments for direct imaging and spectroscopy of exoplanets. HabEx also has two general purpose instruments: a UV imaging spectrograph, and a UV through near-IR imaging spectrograph.

The HabEx prime mission is five years (ten-year design life), with approximately $50 \%$ of the prime mission time dedicated to two ambitious exoplanet surveys a deep survey of eight of our nearest sunlike stars, and a broad survey of roughly 42 nearby, mature stars. The primary difference between the surveys is that the deep survey will systematically search for fainter planets around the very nearest stars, integrating down to a planet-to-star flux ratio detection limit of $2.5 \times 10^{-11}$, which corresponds to a Mars-sized planet around a sunlike star. In comparison, the individual exposure times for the broad survey are set to maximize the overall yield of Earth-like planets and the flux ratio detection limit will generally be higher than in the deep survey. The broad survey is designed to maximize the number of detected and validated EECs. 


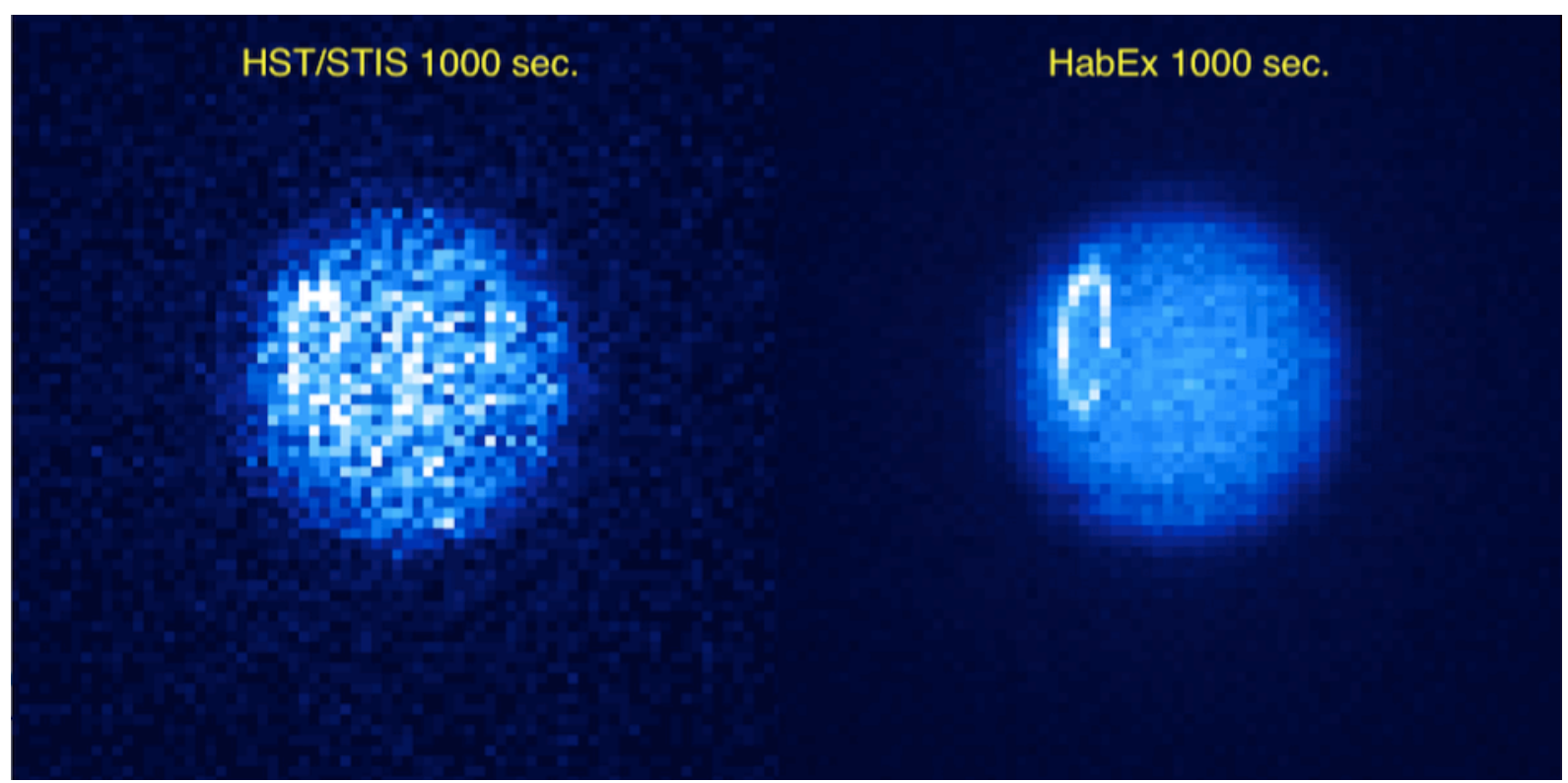

Figure 4. With its high resolution and large effective collecting area in the UV (See Figure 5), HabEx will be able the probe the detailed morphology and time-variable nature of aurorae of the giant planets in our solar system, probing the physics of starplanet interactions. (Left) A simulated image of Uranus with STIS on HST, assuming an exposure time of 1000s. (Right) Same as the left panel, except assuming a 1000s exposure with UVS on HabEx. The larger effective collecting area of HabEx not only enables more detailed studies of the aurorae of Jupiter and Saturn, including changes on timescales of considerably less than an hour, but also enables studies of the morphology and changes of aurorae of Uranus and Neptune on timescales of hours, which is difficult or impossible with HST.

The overall HabEx design has been optimized for high-contrast direct imaging at small angular separations and broad spectroscopy of Earth-sized and larger exoplanets. The off-axis monolithic primary mirror avoids the significant challenges faced by obscured and/or segmented mirrors in achieving both high contrast direct imaging and high planet light throughput with a coronagraph. The Earth-Sun L2 orbit provides a stable thermal and gravitational environment, ideal for high-contrast imaging and formation flying. The dual starlight suppression capabilities provide a flexible approach for optimized exoplanet searches and detailed studies of exoplanets and their planetary systems and is more resilient to uncertainties.

The coronagraph is nimble, residing inside the telescope, allowing for efficient multi-epoch surveying of multiple target stars to identify new exoplanets including EECs, as well as measure their orbits. However, the coronagraph has a narrow annular high-contrast field of view (FOV) with a spectroscopy bandpass limited to $20 \%$, implying that obtaining broadband spectra of detected exoplanets is generally mission-time expensive, as observations must be taken in serial.

Compared to the coronagraph, the starshade provides a wider FOV and broader instantaneous wavelength coverage. However, it is fuel limited rather than target limited due to the thrust required to move the starshade occulter between target stars. For our nominal mission concept, the starshade takes roughly two weeks to travel from one target to another, and only has enough fuel for roughly 100 distinct pointings.

Importantly, this hybrid approach to direct exoplanet detection and characterization is a powerful combination, taking advantage of the complementary strengths of each instrument and significantly increasing the resultant yields of well-characterized planets that have both high-quality broadband spectra and orbits.

\section{THE FOUR HABEX INSTRUMENTS}

\subsection{HabEx Coronagraphic Instrument (HCG).}

The coronagraph mask suppresses starlight from within the telescope to reveal light from proximate exoplanets. HabEx uses a vector vortex coronagraph (VVC) because of its high resilience to common low-order wavefront aberrations, which translates into significantly less stringent requirements on telescope thermal and mechanical stability than other 
coronagraph designs. The HabEx Observatory coronagraph has a 62 mas inner working angle (IWA, representative of the closest detectable exoplanet separation) at $0.5 \mu \mathrm{m}$ with a $20 \%$ bandpass. The coronagraph has a blue channel with a camera and IFS covering $0.45-0.67 \mu \mathrm{m}$, a red channel with a camera and IFS covering $0.67-1.0 \mu \mathrm{m}$, and an IR imaging spectrograph that covers $0.95-1.8 \mu \mathrm{m}$.

\subsection{HabEx Starshade Instrument (SSI).}

The starshade external occulter blocks starlight before it enters the telescope, allowing light from the exoplanet to be observed. The HabEx $52 \mathrm{~m}$ diameter starshade will fly in formation with the telescope at a nominal separation of $76,600 \mathrm{~km}$. The starshade advantages include a high throughput, small IWA, with an outer working angle (OWA) limited only by the instrument FOV and an ultra-broad bandwidth available for high contrast spectroscopy. The HabEx starshade has a 58 milliarcsecond (mas) IWA at $1 \mu \mathrm{m}$ and, a 6 arcsec OWA for broadband imaging, and offers deep enough starlight suppression for spectroscopy over an instantaneous bandwidth of $0.3-1.0 \mu \mathrm{m}$. The starshade may also operate at two additional separations from the telescope. At a larger separation of $115,000 \mathrm{~km}$, it covers bluer wavelengths at $0.2-0.67 \mu \mathrm{m}$ with a constant IWA of 39 mas, providing unique access to deep the ozone features expected in Earth-like atmospheres. At a smaller separation of $42,000 \mathrm{~km}$, it covers redder wavelengths at $0.54-1.8 \mu \mathrm{m}$ with an IWA of 104 mas at $1.8 \mu \mathrm{m}$, enabling sensitivity to multiple water vapor features.

The starshade instrument (SSI) has three channels: a near-UV/blue channel covering $0.2-0.45 \mu \mathrm{m}$ with a grism, a visible channel covering $0.45-0.98 \mu \mathrm{m}$ with an integral field spectrograph (IFS) and camera, and a near-IR channel covering $0.98-1.8 \mu \mathrm{m}$ with an IFS and camera.

\subsection{HabEx UV Spectrograph/Camera (UVS).}

UVS covers $0.115-0.320 \mu \mathrm{m}$ with a FOV of $3 \times 3 \operatorname{arcmin}^{2}$ and multiple spectroscopic settings up to resolutions of 60,000 . Additionally, a grating set contains a mirror to provide imaging capability. The UVS has more than 10 times the effective area of HST's Cosmic Origins Spectrograph (Figure 5) from 160-300nm. Not only does the UVS provide improved angular resolution and throughput relative to HST, it also includes a microshutter array, allowing multiplexed UV slit spectroscopy for the first time in space. Together with the multiplexing capability, UVS will be several orders of magnitude more capable than COS over most of wavelength range of the UVS (115-320nm). The UVS design and science drivers are described in more detail in a companion paper [3].
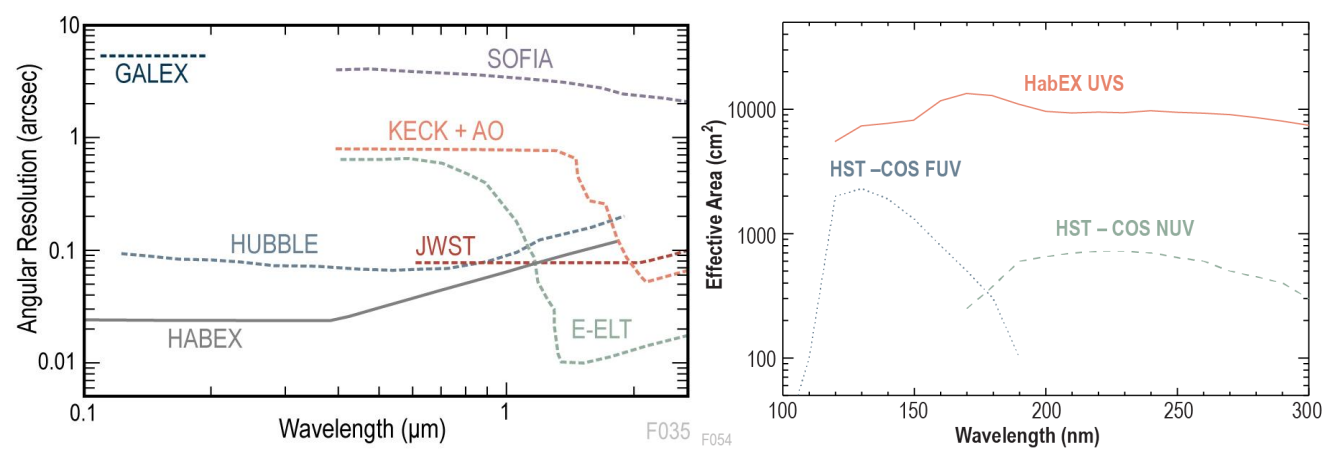

Figure 5. (Left) HabEx will provide the highest-resolution UV/optical images of any current or planned facility, enabling a broad suite of observatory science. Opportunities range from studies of solar system objects, the Milky Way Galaxy, nearby resolved stellar populations, high-redshift galaxies, and large-scale structure. Note we assume that the E-ETL telescope will not successfully achieve extreme adaptive optics, and thus will not be able to achieve their diffraction limit below $\sim 1 \mu \mathrm{m}$. We acknowledge that this assessment this may be pessimistic (Right) With more than ten times the effective area of HSTCOS at wavelengths from 150-300nm, combined with a microshutter array, the HabEx UVS provides several orders of magnitude improved efficiency for UV spectroscopic studies both by the order of magnitude increase in effective area, and multiplexing capability.

\subsection{HabEx Workhorse Camera and Spectrograph (HWC)}

The HWC is an imaging multi-object slit spectrograph with two channels covering wavelengths from the near UV through near-IR and a spectral resolution of 1,000 . The UV/optical channel covers $0.37-0.95 \mu \mathrm{m}$ and the near-IR channel covers $0.95-1.8 \mu \mathrm{m}$. The HWC, with its larger $3 \times 3 \operatorname{arcmin}^{2}$ FOV and higher resolution, will provide capabilities similar to, but significantly more sensitive than, HST's Wide-Field Camera 3 (WFC3) or Advanced Camera for Surveys (ACS). Both the UVS and HWC can be used in parallel with the HCG and SSI instruments. 


\section{HABEX OBSERVATIONAL STRATEGY}

The HabEx exoplanet observational strategy takes advantage of the dual starlight suppression instruments. The broad survey of roughly 42 stars will be undertaken primarily for discovery of small exoplanets. This survey utilizes the coronagraph's pointing agility to revisit the target stars over multiple epochs for discovery, confirmation of physical association with the host star, and measurement semimajor axes to within $25 \%$ and eccentricities to within 0.1 for nearly all detected planets with periods shorter than 10 years.

Broadband spectra of these planetary systems are obtained by the starshade. A deep survey utilizing the starshade for multi-epoch broad bandwidth observations of eight of the nearest sunlike stars will provide even more detailed information about our nearest neighbors, with access to even smaller planets and star-planet separations than in the broad survey.

HabEx does not rely on any prior knowledge or contemporaneous independent observations provided by other ground- or space-based facilities. New observatories, however, are expected to be operational by the time HabEx launches and may provide additional data on the target systems, enabling more robust HabEx target prioritization and scheduling.

Through joint scheduling of exoplanet and general astrophysics observations and engineering design, HabEx is capable of about $90 \%$ observational efficiency. The Guest Observer program will be community driven and competitively selected and will likely include solar system, exoplanet, Galactic, and extragalactic studies.

Two kinds of opportunities will exist to guest observe with HabEx. Standalone observations across the sky will be scheduled during starshade retargeting slews, and concurrent observations utilizing the ability to observe in parallel with the UVS and/or the HWC while observing with any one of the other instruments. Thus, parallel UVS and HWC observations will enable images and/or spectra in two separate $3 \times 3 \operatorname{arcmin}^{2}$ FOVs during observations by the SSI or HCG, providing, e.g., two HST-like ultra-deep fields in the vicinity of the exoplanet target stars. Similarly, the HWC can obtain parallel observations during observations by the UVS, and vice versa. Thus, the four instruments on HabEx are highly multiplexed, thereby greatly improving the mission's scientific productivity.

The distribution of observing time for all HabEx programs is shown in Figure 6.

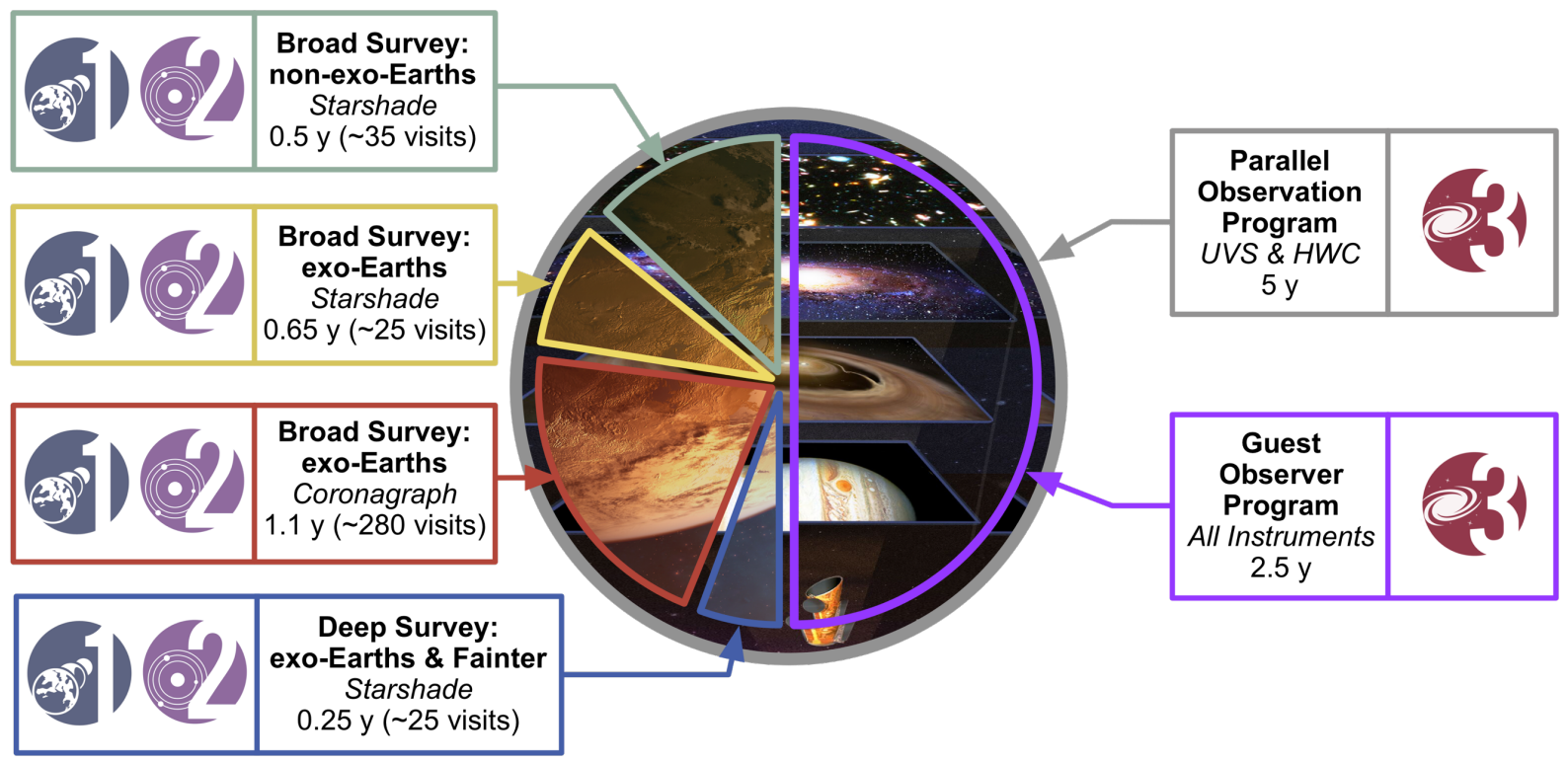

Figure 6. HabEx is an observatory for the greater astrophysics community, balancing exoplanet and general astrophysics observing programs. All of the instruments can be operated in parallel, and thus HabEx is also capable of parallel deep field observations with one or both general astrophysics instruments during the entire mission duration.

\section{HABEX PHILOSOPHY}

HabEx was designed to be a Great Observatory that can be realized in the 2030s. To achieve this, the guiding philosophy of this study was the recognition that any recommendation by the Astro2020 Decadal Survey must balance 
scientific ambition with programmatic and fiscal realities, while simultaneously considering the impact of its development schedule on the greater astronomy community and the need for a broad portfolio of science investigations. The HabEx study therefore aimed to develop a mission capable of the most compelling science possible, while still adhering to likely cost, technology, risk, and schedule constraints.

The preferred HabEx architecture was thus chosen to be technically achievable within this time frame, leveraging the maturation of several enabling technologies over the last decade or more. HabEx adopted a conservative design with substantial margins, utilizing moderate to high technological maturity resulting in low development risk. We discuss this conservative approach to both adopting high TRL technologies and making the design as simple as possible to lower risk and cost in more detail in the scientific and technological readiness in an accompanying paper [1]. HabEx also provides the community with imaging and spectroscopic capabilities orders of magnitude better than Hubble, which uniquely compliments currently-planned space and ground based observatories.

Finally, this study also considers eight other architectures, which offer greater flexibility in budgeting and phasing, such that HabEx may still be compatible with a balanced portfolio, even for the most pessimistic fiscal projections. Nevertheless, all nine architectures still enable groundbreaking science. Thus, this study provides the Astro2020 Decadal Survey additional flexibility in its decision making.

HabEx is ambitious, offering humankind the first opportunity a glimpse into worlds like our own and uncover the signs of life. It provides a Hubble-like platform to support community science with deep, high resolution, multiwavelength observations. As one of four large strategic missions under consideration by the Decadal, HabEx recognizes that any recommendation by the Decadal will have to balance scientific ambition with the programmatic and fiscal realities that constrain space exploration. HabEx also recognizes the impact of prolonged development programs to the greater astronomy community, as demonstrated by James Webb Space Telescope, that limit the ability of NASA to fund a broad portfolio of science investigations concurrent with mission development. From the beginning, the HabEx mission was guided by a philosophy to offer the most compelling science case as constrained by the realities each large strategic mission concept is beholden to.

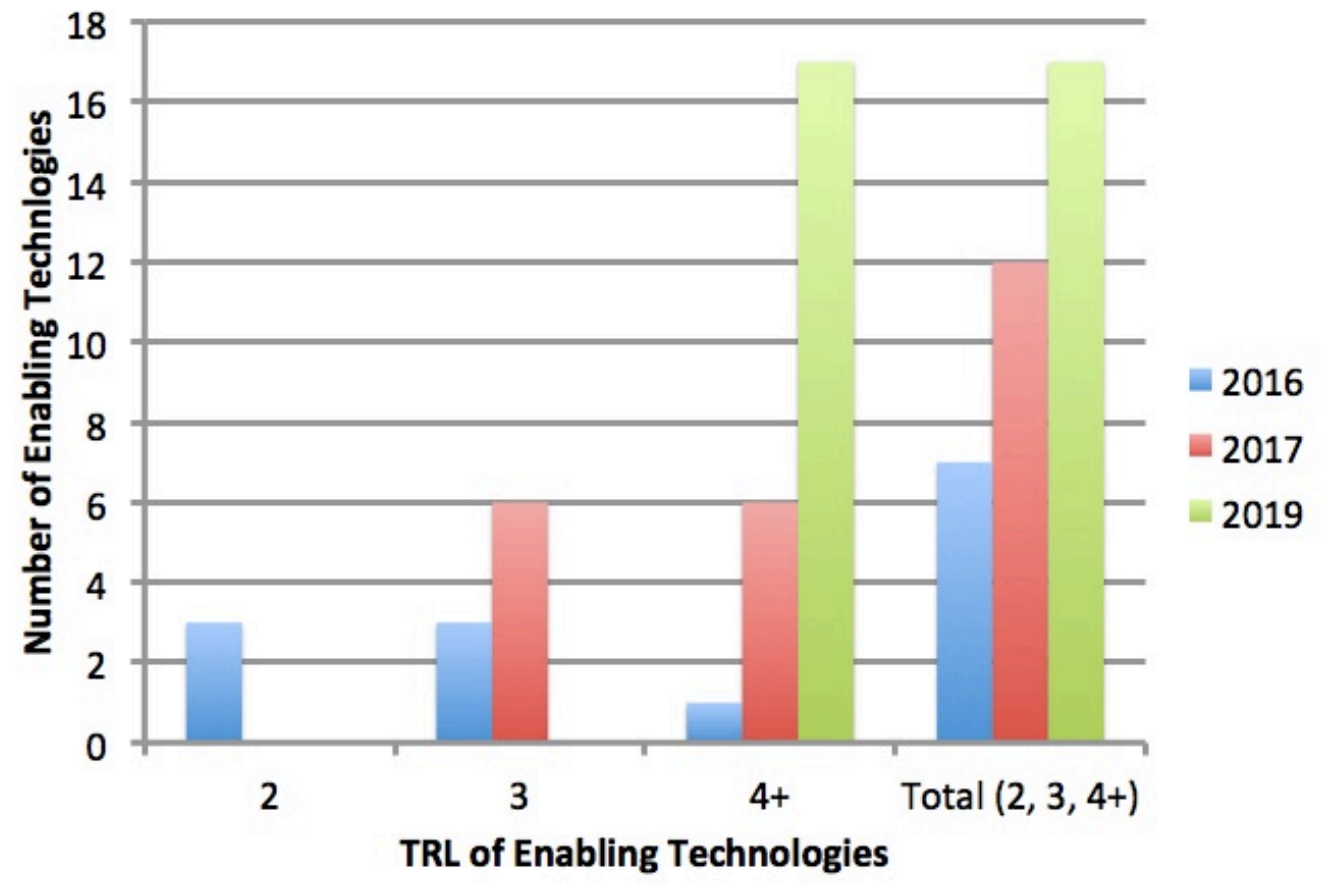

Figure 7. Development of the number of TRL 2, 3 and 4+ technologies over the course of the last three years of the HabEx mission study. There are two important points to note. First, all TRL 2 and 3 technologies have been matured to TRL 4 or higher. Second, the total number of self-identified technologies that HabEx is carrying has increased from 7 in 2016, to 12 in 2017, to 17 currently. This is primarily due to the fact that, as the concept design matured, the HabEx team recognized that items that had been identified as one technology were really several independent technologies. It is not because our design has become significantly more complex since 2016. Only one completely new technology (microthrusters) was added between 2016 and today. 


\section{HABEX ALTERNATE DESIGNS AND ARCHITECTURE TRADES}

HabEx has evaluated the comparative science yield and cost for nine architectures in order to support the Decadal Survey's assessment of the optimal implementation to achieve HabEx's science. The primary HabEx architecture is a $4 \mathrm{~m}$ monolithic telescope employing both coronagraph and starshade high-contrast imaging technologies. It is the preferred HabEx option and has been studied in depth to develop an accurate cost estimate and verify that it is responsive to all science objectives in the science traceability matrix.

Beyond the HabEx baseline 4m telescope configuration, HabEx has two distinct well-vetted options that enable an alternative mission at a lower cost but with reduced science capabilities and yield. The first is a coronagraph-only option using the same $4 \mathrm{~m}$ off-axis telescope as in the baseline design, launching in an SLS launch vehicle. The second is a starshade-only option with a $3.2 \mathrm{~m}$ segmented-mirror telescope, this system would fit into a single Falcon Heavy launch vehicle. See [5] for a detailed description of one of the second design.

To reach the baseline and alternate designs, the STDT considered a trade of six additional options, three telescope mirror diameters $(4 \mathrm{~m}, 3.2 \mathrm{~m}$, and $2.4 \mathrm{~m})$ for three different configurations of starlight suppression: hybrid starshadecoronagraph, starshade only, and coronagraph only. We have evaluated whether each of the nine architectures meet the baseline or threshold requirements of each of the 17 specific science objectives that were designed to set the requirements needed to achieve HabEx's three primary science goals.

All $2.4 \mathrm{~m}$ options were considered less compelling due to their low planet discovery yield and limited capability for observatory science. It should be emphasized, however, that although the $2.4 \mathrm{~m}$ designs are not able to detect and characterize a significant number of EECs, they are able to detect and characterize a significant number of larger planets, and thus would provide an excellent dataset for comparative exoplanetology. And, of course, these missions have the same aperture as HST, which is still enabling groundbreaking science after nearly 30 years after its launch. Notably, the oversubscription rate of HST shows no evidence of a significant secular decline over its nearly 30 -year lifetime.

\section{WHY NOW? SCIENTIFIC READINESS}

HabEx stands available as an achievable mission to directly image exo-Earths, building upon decades of scientific and technological achievement. There has been tremendous progress in the discovery of exoplanets over the last 20 years. In particular, astronomers have discovered that small rocky planets around main sequence stars are common. One implication of these discoveries is the prospect for atmospheric characterization of rocky planets orbiting M dwarf stars in the near-term. These systems have been and will be identified by surveys such as MEarth [5], TRAPPIST/SPECULOOS [6], and NASA's Transiting Exoplanet Survey Satellite (TESS) [7]. The atmospheres of these planets will be characterized by follow-up observations from ground-based ELTs and space-based missions such as JWST and eventually even HabEx. In parallel, steady progress in high-contrast direct imaging technology has been very impressive, with the first direct detection of bright self-luminous exoplanets announced in 2008 (e.g., [8]), and the characterization of closer-in selfluminous planets since the (e.g., [9])

This progress points to the next logical step: the discovery and detailed characterization of Earth-like worlds and complete planetary systems around nearby sunlike stars. We now know, thanks primarily to NASA's Kepler mission, that small planets orbiting sunlike stars are not rare. Indeed, although difficult to measure and requiring some extrapolation, we know that the frequency of rocky planets in the habitable zones of sunlike stars is likely not very small, nor very large. The best estimates indicate that $\sim 25 \%$ of all sunlike stars host a rocky planet at the right distance to its host star to have liquid water [1], assuming appropriate atmospheric conditions.

HabEx will start this journey of exploration, providing the first detailed images and spectra of the full range of exoplanets orbiting nearby mature stars, and searching for signs of habitability and life on all of the small rocky worlds detected.

\section{WHY NOW? TECHNOLOGICAL READINESS}

The preferred HabEx architecture was thus chosen to be technically achievable within the 2035 timeframe, leveraging the maturation of several enabling technologies over the last decade or more. HabEx adopted a conservative design with substantial margins, utilizing moderate to high technological maturity resulting in low development risk.

The HabEx Observatory design is based on technologies that are at or near state of the art with clear paths of

development. These technologies are being developed by existing teams with existing staffing. The design favors high TRL 
technologies as a strategy to minimize development risk and reduce potential cost. All enabling technologies are at TRL 4 or higher, with many expected to be at TRL 5 or higher by 2023 under currently funded development.

Through careful design choices, lessons learned from past studies (particularly the Exo-Starshade and ExoCoronagraph probe studies), and utilization of past and ongoing investments into these technologies, the preferred HabEx architecture design minimizes cost and risk, while maximizing scientific return. HabEx's enabling technologies are discussed in more detail in a companion paper [4].

\section{BEGINNING A NEW ERA OF ASTROPHYSICS WITH HABEX}

HabEx is a cost-effective, modest risk, high-impact science mission. HabEx will leverage recent advancements in starlight suppression technologies to utilize both a coronagraph and starshade to seek new worlds and explore their habitability and map our nearest neighbor planetary systems to understand the diversity of the worlds they contain.

While the HabEx mission architecture is capable of direct imaging and spectral characterization of a broad range of exoplanets, including Earth analogs orbiting nearby sunlike stars, HabEx also provides unique capabilities for UV through near-IR astrophysics and solar system science from the vantage of space, moving UV capabilities to the next level after HST retires. HabEx is a worthy UV/optical successor to HST in the 2030s with significantly improved sensitivity and spatial resolution stemming from HabEx's significantly larger 4m diameter aperture, improved detector technology, exquisite wavefront control, and a more thermally stable orbit.

\section{ACKNOWLEDGEMENTS}

Part of this research was carried out at the Jet Propulsion Laboratory, California Institute of Technology, under a contract with the National Aeronautics and Space Administration.

\section{REFERENCES}

[1] Kopparapu, R., et al., "Exoplanet Classification and Yield Estimates for Direct Imaging Missions," Astrophys. $J ., 856,122(2018)$

[2] Stahl, H.P., "HabEx baseline telescope design and predicted performance," Proc. SPIE (2019).

[3] Scowen, P.A, et al. "The HabEx Ultraviolet Spectrograph (UVS): design and science drivers," Proc. SPIE (2019).

[4] Redding, D.C., "A Habitable Exoplanet Observatory (HabEx) starshade-only alternative," Proc. SPIE (2019).

[5] Charbonneau, D., et al., "A super-Earth transiting a nearby low-mass star," Nature, 462, 891 (2009)

[6] Gillon, M., et al., "Seven temperature terrestrial planets around the nearby ultracool dwarf star TRAPPIST-1," Nature, 542, 446 (2017)

[7] Ricker, G., et al., "The Transiting Exoplanet Survey Satellite,” JATIS, 1, 014003 (2015)

[8] Marois, C., et al., "Direct Imaging of Multiple Planets Orbiting the Star HR 8799," Science, 322, 5906 (2008)

[9] Macintosh, B., et al., "Discovery and Spectroscopy of the young jovian planet with the Gemini Planet Imager," Science, 350, $6256(2015)$ 